\title{
Discrimination tests of visually influenced syllables
}

\author{
LAWRENCE D. ROSENBLUM and HELENA M. SALDANA \\ University of California, Riverside, California
}

\begin{abstract}
In the McGurk effect, perception of audiovisually discrepant syllables can depend on auditory, visual, or a combination of audiovisual information. Under some conditions, visual information can override auditory information to the extent that identification judgments of a visually influenced syllable can be as consistent as for an analogous audiovisually compatible syllable. This might indicate that visually influenced and analogous audiovisually compatible syllables are phonetically equivalent. Experiments were designed to test this issue using a compelling visually influenced syllable in an AXB matching paradigm. Subjects were asked to match an audio syllable / $/ \mathrm{va}$ either to an audiovisually consistent syllable (audio / $/ \mathrm{va}$-video /fa/) or an audiovisually discrepant syllable (audio $/ \mathrm{ba}$-video /fa/). It was hypothesized that if the two audiovisual syllables were phonetically equivalent, then subjects should choose them equally often in the matching task. Results show, however, that subjects are more likely to match the audio /va/ to the audiovisually consistent $/ \mathrm{va}$ /, suggesting differences in phonetic convincingness. Additional experiments further suggest that this preference is not based on a phonetically extraneous dimension or on noticeable relative audiovisual discrepancies.
\end{abstract}

Much attention has recently been given to the perception of audiovisually presented syllables (see Summerfield, 1987, for a review). This is partly because of the interesting phenomenon known as the "McGurk effect" (McGurk \& MacDonald, 1976). In one strong version of the effect, the auditory syllable $/ \mathrm{ba} /$ is repeatedly dubbed onto a video tape of a speaker's face producing the syllables /be/, /ve/, /de/. Upon seeing this dubbed video tape, observers report hearing the syllables $/ \mathrm{ba} /, / \mathrm{va} /, / \mathrm{da} /$ (Liberman \& Mattingly, 1985). The effect is quite striking: even when completely aware of the dubbing procedure, observers still report hearing a clearly defined syllable influenced by what they see. Furthermore, in this strong form of the effect, observers cannot distinguish the auditory and visual components of the resultant perception, that is, they cannot tell that the initial consonants are given visually while the vowels are given auditorily (Liberman \& Mattingly, 1985; see also Massaro, 1987). Consequently, the McGurk effect has relevance to general theories of speech perception and the question of the perceptual object of speech (Diehl \& Kluender, 1989; Fowler \& Rosenblum, 1991; Liberman \& Mattingly, 1985; Massaro, 1987).

Although the McGurk effect works better with some audiovisual syllable combinations than with others, when the effect works, it seems to produce compelling visually influenced percepts. For example, subjects often report

This research was supported by an Intramural Grant from the University of California. We gratefully acknowledge the assistance of Kathleen Gurskis, Theresa Osinga, and Paige Wuestefeld as well as the comments of Carol Fowler. Requests for reprints should be sent to Lawrence Rosenblum, Department of Psychology, University of California, Riverside, California 92521-0426. hearing the visually influenced syllable over $95 \%$ of the time-competitive with identification results of analogous audiovisually compatible syllables (Repp, Manuel, Liberman, \& Studdert-Kennedy, 1983). Also, the effect works well with both consonant and vowel components of consonant-vowel (CV) syllables (Summerfield \& McGrath, 1984) as well as with some words (see Summerfield, 1987). The effect also occurs with computer-generated visual face stimuli of both detailed (Massaro \& Cohen, 1990) and schematic nature (Summerfield, Macleod, McGrath, \& Brooke, 1989). Finally, recent results indicate that the McGurk effect occurs even when the auditory and visual components are of speakers of different genders (Green, Kuhl, Meltzoff, \& Stevens, 1991). This last observation is particularly striking considering that in such instances, subjects are completely aware that the audio and visual components were provided by different speakers.

Taken together, these results might imply that a compelling visually influenced syllable can be as phonetically convincing as its audiovisually compatible counterpart. In other words, visually influenced and audiovisually compatible syllables might be phonetically indistinguishable. However, most studies of McGurk effects have involved only identification judgments and have therefore only revealed what subjects generally hear. Few tests of the details of subjects' phonetic impressions have been made with incompatible audiovisual stimuli (however, see Massaro, 1987, as discussed below).

To determine whether visually influenced syllables are phonetically equivalent to audiovisually compatible syllables, a more fine grained test of perceptual equivalence could be used. Often, such tests involve a discrimination or matching task (cf. Liberman, Harris, Hoffman, \& 
Griffith, 1957). In an AXB matching task, for example, subjects are given the choice to match the $\mathrm{X}$ stimulus to either stimulus A or B. For audiovisual stimuli, an example of an AXB presentation could include $A$, a visually influenced syllable made up of audio $/ \mathrm{ba} /$-visual $/ \mathrm{va} / ; \mathrm{X}$, an auditory-only $/ \mathrm{va} /$; and $\mathrm{B}$, an audiovisually compatible syllable made up of audio /va/-visual / va/. Subjects would then be asked to match the $X$ token to either the A or B tokens, basing their judgments only on what they hear (using an $X$ token that is auditory only would help to ensure this). If the visually influenced syllable is phonetically equivalent to its audiovisually compatible counterpart, then it would be predicted that subjects should match $\mathrm{X}$ to $\mathrm{A}$ and $\mathrm{B}$ with equal frequency.

Experiments using audiovisual stimuli in a discrimination paradigm (same-different judgments) have been reported by Massaro (1987). However, the goal of those experiments was to determine the degree of perceptual equivalence between various audiovisually discrepant stimuli. Thus, no matching tests of phonetic equivalence for audiovisually discrepant and consistent stimuli have been conducted.

The question of phonetic equivalence was tested in Experiments 2 and 3 of this paper using a compelling visually influenced / $/ \mathrm{va}$ / syllable. The AXB sequence outlined above was implemented for these experiments. Experiment 1 used a somewhat different AXB sequence to test whether subjects would match a visually influenced $/ \mathrm{va} /$ (audio /ba/-video /va/) to either an audio /ba/ or an audio /va/. This design addresses the question of whether the audio $/ \mathrm{ba} /$-video $/ \mathrm{va} /$ is sufficiently phonetically convincing to be heard as / va/ while both an auditory /ba/ and $/ \mathrm{va} /$ are immediately present.

Given the aforementioned strength of McGurk-type effects, it could be predicted that our compelling visually influenced /va/ should be sufficiently phonetically convincing so as to elicit a match to audio /va/ in Experiment 1 and to be chosen to match an audio /va/ equally often as an audiovisually compatible / va/ in Experiments 2 and 3.

\section{Preparation of Audiovisual Stimuli}

To find the most compelling visually influenced syllable, a number of different audiovisual syllable combinations were recorded and dubbed and then used in informal identification tests. The recordings were of an AmericanEnglish-speaking male actor producing the syllables $/ \mathrm{fa} /$, /ra/, /ga/, /da/, /la/, /na/, /ma/, //a/, / /a/, /ta/, /ba/, /pa/, and $/ \mathrm{va} /$. The actor was instructed to keep both the intonation and the duration of each syllable constant. A Panasonic PVS350 camcorder and a Shure SM57 microphone were used to record the initial stimulus tape. The actor was seated $5 \mathrm{ft}$ in front of the camera. His head was placed in a wire headbrace to inhibit movement. The camera was centered on the actor's lips. The recorded image consisted of the bottom of the actor's nose to the bottom of his chin; no background was visible. Each syllable was recorded four times on the initial tape.
A Compaq 386/25 computer and two Panasonic video recorders were used for dubbing the audiovisual tokens. The original audio tokens were input into files in the computer. These tokens were then combined with various discrepant video tokens in informal pilot experiments so that we could determine which audiovisual pair demonstrated the greatest visual influence. The most convincing discrepant pairing was an audio $/ \mathrm{ba} /$ dubbed onto a video $/ \mathrm{fa} /$, which resulted in a clear percept of $/ \mathrm{va} /$. (This is consistent with the identification results reported by Repp et al., 1983.) To use these stimuli in an AXB matching context, it was important that both the /va/ and the /ba/ audio tokens had the same general acoustic characteristics (e.g., intensity, fundamental frequency contour). Accordingly, an additional file was made by editing the audio /va/ syllable until it sounded like a /ba/. This was accomplished by deleting the initial $55 \mathrm{msec}$ of the / $\mathrm{va} /$ token. It was determined through pilot experiments that this hybrid /ba/ was the most susceptible token to a visual influence that could still elicit consistent / $\mathrm{ba} /$ identifications on its own.

The / $/ \mathrm{a} /$ and hybrid /ba/ files were output to a second videotape whenever a voice-activated circuit was closed. The output of the audio channel from the original tape served as the input to this circuit so that when an audio token from this original tape was produced, a stored token was immediately output to the second tape. Simultaneously, the video token of the original tape was recorded onto the second tape, resulting in a synchronous audiovisual token. The lag time for dubbing was found to be no greater than $9.4 \mathrm{msec}$, well below the 80 -msec range required for observers to detect an audiovisual asynchrony (McGrath \& Summerfield, 1985).

\section{EXPERIMENT 1}

The first experiment was designed to determine whether the subjects would match a visually influenced / va/ (audio /ba/-video /fa/) to an audio /va/ or an audio /ba/. Given past findings using identification judgments, it was predicted that the subjects would hear the visually influenced syllable as /va/ and match it to the audio /va/. To ensure that the audiovisual stimulus was a good candidate for this test, identification judgments were also collected for the visually influenced syllable.

\section{Method}

Stimuli. The audio stimuli for the first experiment consisted of the aforementioned $/ \mathrm{va} /$ token and the $/ \mathrm{ba} /$ token that was edited from it. The visual stimulus consisted of a $/ \mathrm{fa} /$. These tokens were arranged into AXB triads in the following manner: The A and B stimuli were audio only and were always different from one another. The $\mathrm{X}$ stimulus was either a consistent audiovisual token (audio $/ \mathrm{va} /$-video /fa/) or a discrepant audiovisual token (audio /ba/-video $/ \mathrm{fa} /$ ). An example of a critical triad is shown schematically in Figure 1. Four triads were produced overall. Two triads included the audiovisually discrepant $\mathbf{X}$ token and differed only in whether the audio / va/ or audio / $\mathrm{ba} /$ was in the A position. The other two triads included the audiovisually compatible $\mathrm{X}$ token and differed only 


\begin{tabular}{|c|c|c|}
\hline Audio & $\frac{\mathrm{A}}{\mathrm{va}}$ & $\underset{\mathrm{Da} /}{\mathrm{X}}$ \\
\hline Video & & $/ \mathrm{fa} /$ \\
\hline
\end{tabular}

Mean \% Matches

Mean \% Correct Identifications (based on audio portion of stimulus)

Figure 1. Example of a critical AXB matching condition used in Experiment 1 with mean percentage results of critical conditions.

in whether audio / va/ or audio / ba/ was in the A position. This second set served as a control and, additionally, served to keep the subjects from becoming discouraged if they found the critical triad sets too difficult.

Each audiovisual token lasted $2 \mathrm{sec}$, allowing for the entire articulatory event to be seen. (The audio portions of the presentations lasted $621 \mathrm{msec}$ for the /va/ token and $566 \mathrm{msec}$ for the / $/ \mathrm{ba} /$ token.) Accordingly, the time between onsets of each token in the triads was $2 \mathrm{sec}$, producing triads of $6 \mathrm{sec}$ total. For the audio-alone tokens of the triads, the video image was black. The switch from a video image to video black was instantaneous. The four triads were recorded 10 times each in random order. An interstimulus interval (ISI) of $4 \mathrm{sec}$ (consisting of silence and video black) was placed between triads.

The same stimulus set was also presented with the video portion of the presentations switched off. This condition served as an additional control in order to isolate the influence of visual information.

The presentation tape also included audio-alone, video-alone, and audiovisual identification stimuli. The audio-alone identification stimuli consisted of 10 audio / va/ tokens, and 10 audio $/ \mathrm{ba} /$ tokens (edited from $/ \mathrm{va} /$ ), which were recorded in random order with an ISI of $3 \mathrm{sec}$. The video identification stimuli consisted of 10 video /fa/tokens, $10 \mathrm{video} / \mathrm{va} /$ tokens, and $10 \mathrm{video} / \mathrm{ba} /$ tokens, which were recorded in random order with an ISI of $3 \mathrm{sec}$. The stimuli for the audiovisual identification consisted of the following three tokens: an audio $/ \mathrm{ba} /$-video / $\mathrm{fa} /$, an audio $/ \mathrm{va} /$-video $/ \mathrm{fa} /$, and an audio $/ \mathrm{ba} /$-video $/ \mathrm{ba}$. The first two tokens were presented 10 times each, and the third token was presented 20 times. (Twice as many consistent /ba/ tokens were used since pilot research had shown that most subjects would perceive both the consistent and discrepant $/ \mathrm{va}$ / tokens as $/ \mathrm{va} /$, producing roughly $20 / \mathrm{va} /$ responses. Thus, using 20 consistent $/ \mathrm{ba} /$ tokens would act to guard against response bias.) These tokens were also recorded in random order with an ISI of $3 \mathrm{sec}$.

The conditions' presentation ordering was counterbalanced across subject groups as specified in the Procedure.

Subjects. Fourteen undergraduates at the University of California, Riverside, participated in the experiment for partial credit of a class requirement. All subjects reported having normal or corrected-tonormal vision and normal hearing.

Procedure. The subjects were run in groups of 2 or 3 . To monitor the potential effects of presentation ordering, different subject groups received different condition orderings. Six subjects received the conditions in the order: audiovisual AXB, audio-alone AXB, audiovisual identification, video identification, and audio identification. Three subjects received the conditions in the order: audioalone AXB, audiovisual AXB, audiovisual identification, video identification, and audio identification. Three subjects received the conditions in the order: audiovisual identification, audiovisual AXB, audio-alone $\mathrm{AXB}$, video identification, and audio identification. Two subjects received the conditions in the order: audiovisual identifi- cation, audio-alone $\mathrm{AXB}$, audiovisual $\mathrm{AXB}$, video identification, and audio identification.

The subjects were instructed that they would be presented with audiovisual and audio-only tokens of a speaker producing different syllables. They were told that during the experiment, they would be performing one of two types of judgments on the syllables. If asked to identify the tokens, they were simply to write down the syllables they perceived on an answer sheet. If asked to match syllables, they would hear a set of three syllables and then indicate whether the middle syllabie of the three sounded more like the first or third. If the middle syllable seemed more like the first, they were to write an $A$ on the answer sheet, and if the middle syllable seemed like the third, they were to write a $B$ on the answer sheet. They were told that it was important that they attend to all three syllables before making their judgments. In addition, they were told to look back up at the video monitor as soon as they completed each judgment. The experimenter watched the subjects throughout the experiment to ensure that they did see each presentation in its entirety. If a subject needed more time to finish a judgment than the allotted ISI, the experimenter paused the videotape and waited for the subject to look back up at the monitor.

The subjects were informed that about half of the trials would involve audiovisual presentations of the syllables. They were told that for these trials, even though they should both watch and listen to the stimuli, it was important that they base their identifications and matching judgments only on what they heard.

The subjects were shown two examples of the matching task before the experiment actually began. These examples were audiovisual control triads, allowing the subjects to successfully match the $\mathrm{X}$ token to one of the $\mathrm{A}$ or $\mathrm{B}$ tokens. The visual stimuli were presented on a 19-in. color monitor situated $8 \mathrm{ft}$ away from the subjects. The audio stimuli were presented through a speaker placed directly below the video monitor and were presented at a comfortable listening level. The subjects sat about $3 \mathrm{ft}$ apart from one another and were monitored to ensure that they were not basing their judgments on a neighboring subject's responses. The experiment lasted approximately $1 \mathrm{~h}$

\section{Results and Discussion.}

Pooled results for certain critical conditions are shown in Figure 1.

The discrepant audiovisual identification trials were analyzed first to determine if our audio / $\mathrm{ba} /$-video / fa/token could truly be considered a compelling visually influenced syllable. The mean percentage of "correct" responses (responses for which the McGurk effect did not occur and the subjects reported hearing the audio token, /ba/) for each subject can be seen in column 2 of Table 1. The average percentage correct across subjects was $4 \%$ (see 
Table 1

Percentage of Correct Responses per Subject for Audiovisual Identification, Audiovisual Discrimination, and Audio-Alone Discrimination for Experiment 1

\begin{tabular}{cccc}
\hline Subject & Audiovisual ID & Audiovisual AXB & Audio-Alone AXB \\
\hline 1 & 0 & 25 & 80 \\
2 & 0 & 15 & 85 \\
3 & 0 & 25 & 80 \\
4 & 0 & 50 & 85 \\
5 & 20 & 50 & 90 \\
6 & 0 & 50 & 100 \\
7 & 0 & 30 & 100 \\
8 & 0 & 20 & 100 \\
9 & 0 & 0 & 95 \\
10 & 0 & 70 & 100 \\
11 & 0 & 40 & 100 \\
12 & 10 & 5 & 100 \\
13 & 20 & 40 & 100 \\
14 & 10 & 35 & 100 \\
$M$ & 4.3 & 32.5 & 93.9 \\
$S D$ & 7.6 & 19.3 & 8.1 \\
\hline
\end{tabular}

Figure 1), which is roughly equivalent to percentages reported elsewhere for similar stimuli (e.g., Repp et al., 1983). Thus, the subjects reported hearing the syllable $/ \mathrm{va} / 96 \%$ of the time when presented with an audio /ba/-video /fa/, suggesting that it was an appropriate audiovisual presentation for testing in the discrimination experiments. The percentage correct for the consistent / $\mathrm{ba} /$ audiovisual identification token was $100 \%$, and the percentage correct for the consistent / $\mathrm{va} /$ audiovisual identification (audio /va/-video /fa/) was $92 \%$ (1 subject responded with $/ \mathrm{da} / \mathrm{s}$ instead of $/ \mathrm{va} / \mathrm{s}$ ). The mean percentages correct for the audio-alone identifications were $99 \%$ for the $/ \mathrm{va} /$ token and $95 \%$ for the $/ \mathrm{ba} /$ token.

Finally, the mean percentages for the video-alone identifications were calculated for each response type. For the $/ \mathrm{ba} / \mathrm{video}$ token, there were $96 \%$ correct $/ \mathrm{ba} /$ responses and $4 \% / \mathrm{va} /$ responses. For the $/ \mathrm{va} /$ token, there were $84 \%$ correct $/ \mathrm{va} /$ responses, $10 \% / \mathrm{ba} /$ responses, and $6 \%$ responses other than $/ \mathrm{va} /, / \mathrm{ba} /$, or $/ \mathrm{fa} /$. Finally, for the $/ \mathrm{fa} /$ video token, there were $0 \%$ correct $/ \mathrm{fa} /$ responses, $92 \% / \mathrm{va} /$ responses, $2 \% / \mathrm{ba} /$ responses, and $7 \%$ responses other than $/ \mathrm{va} /, \mathrm{ba} /$, or $/ \mathrm{fa} /$. Thus, the $/ \mathrm{fa} /$ visual token was identified most often as /va/. The fact that the subjects identified the visual $/ \mathrm{fa} / \mathrm{as} / \mathrm{va} /(\mathbf{9 2} \%)$ more often than the visual $/ \mathrm{va} / \mathrm{as} / \mathrm{va} /(84 \%)$ might explain why we found the $/ \mathrm{fa} /$ to be more impressive in influencing a heard /va/.

The AXB discrimination trials were then analyzed using the same criterion for determining a correct response (defined as a match based on audio only). The subjects' percentages of correct responses for the critical audiovisual AXB triads are listed in column 3 of Table 1 . The pooled mean percentage (across subjects) for correct judgments was $33 \%$ (see Figure 1). Using a $t$ test, it was determined that the percentage of correct judgments was significantly less than chance $[t(13)=3.39, p<.05]$. This suggests that on average, the subjects were more likely to match the visually influenced / $\mathrm{va} /$ to the audio / $\mathrm{va} /$ than to the audio $/ \mathrm{ba} /$. There was no effect of stimulus presentation ordering on responses for the critical audiovisual AXB triads $[F(3,10)=2.869, p>.05]$. The subjects' percentages of correct responses for the critical audio-alone AXB triads are listed in column 4 of Table 1 . The percentage correct for the critical audio-alone AXB triads across subjects was found to be $94 \%$, which was significantly greater than chance $[t(13)=20.221, p<.05]$. Using a twotailed $t$ test, the percentage correct for the critical audiovisual $\mathrm{AXB}$ triads was compared to the percentage correct for the audio-alone AXB triads and was found to be significantly different $[t(13)=11.58, p<.05]$. This suggests that there was a significant influence of visual information for the critical AXB triads. The percentage correct for the audiovisual control triads was $92 \%$.

Thus, on average, the subjects reported hearing the visually influenced / $\mathrm{va} /$ in both identification and matching contexts. It should be noted that they do so with greater frequency in the identification task $(96 \%)$ than in the matching task $(67 \%)$. Although the reasons for this difference are unclear, it could be that presenting the audioalone stimuli so close in time to the target audiovisual token allowed the subjects to concentrate on what was heard somewhat more easily in the matching task. If so, the subjects could be making their matches on the basis of either relevant or extraneous phonetic properties (see below). This issue will be addressed in Experiments 2 and 3.

The overall outcome of Experiment 1, however, shows that for these stimuli, the McGurk effect does work in an AXB discrimination context. In the next series of experiments, we address the question of whether a visually influenced syllable is phonetically equivalent to an audiovisually compatible syllable.

\section{EXPERIMENT 2}

In Experiment 2, the AXB methodology was used to determine if a visually influenced syllable is phonetically equivalent to its audiovisually compatible counterpart. 
(A)

$\begin{array}{lccc} & \underline{\mathrm{A}} & \underline{\mathrm{X}} & \underline{\mathrm{B}} \\ \text { Audio } & / \mathrm{ba} / & / \mathrm{va} / & / \mathrm{va} / \\ \text { Video } & / \mathrm{fa} / & & / \mathrm{fa} / \\ & & & 78 \\ \text { Mean \% Matches } & 22 & 100 \\ \begin{array}{l}\text { Mean \% Correct Identifications } \\ \text { (based on audio portion }\end{array} & 21 & & \end{array}$

(B)

$\underline{\mathrm{A}}$

Audio

Video

Mear \% Matches

Mean $\%$ Correct Identifications (based on audio portion of stimulus) /ba/

/fa/

23

5
B

$/ \mathrm{va} /$

$/ \mathrm{fa} /$

Figure 2. Example of a critical AXB matching condition used in Experiment 2 with mean percentage results of critical conditions for (A) all subjects and (B) subjects who show a strong McGurk effect.

Given the success of our visually influenced /va/ stimulus in Experiment 1, this token was used again in Experiment 2 . The critical triads took the form of an audio-alone $/ \mathrm{va} /$ in the $\mathrm{X}$ position, an audio / $\mathrm{ba} /$-video / $\mathrm{fa} /$ in either the $A$ or $B$ position, and an audio $/ \mathrm{va} /$-video $/ \mathrm{fa} /$ in the remaining $\mathrm{A}$ or $\mathrm{B}$ position. (Using an audio-alone $/ \mathrm{va} /$ in the $\mathrm{X}$ position should help the subjects base their matching judgments on what they hear.) An example of a critical triad is shown schematically in Figure 2 . If the visually influenced $/ \mathrm{va} /$ is phonetically equivalent to the audiovisually compatible /va/, then the subjects should be equally likely to match the two audiovisual syllables to the audio-alone /va/.

\section{Method}

Stimuli. The stimuli for Experiment 2 consisted of the original audio / $\mathrm{va} /$ token, the $/ \mathrm{ba} /$ token that was edited from it, and video $/ \mathrm{fa} /$ and $/ \mathrm{ba} /$. The AXB triads were arranged in the following way: The $X$ token was always an audio-alone $/ \mathrm{va} /$. The $A$ and $B$ tokens were audiovisual and always different from each other. For the two critical triads, they were a discrepant token (audio $/ \mathrm{ba} /$-video $/ \mathrm{fa} /$ ) and a consistent token (audio/va/-video/fa/). The two critical triads differed only in whether the audiovisually compatible token was in the $A$ or $B$ position. For the two control triads, the $A$ and $B$ tokens were both consistent audiovisually (audio $/ \mathrm{ba} /$-video $/ \mathrm{ba} /$, audio $/ \mathrm{va} /$-video $/ \mathrm{fa} /$ ) and differed only in which of the two tokens was in the A position. In addition, four other triads were produced that were of no relevance to the current experiment. The eight different triads were recorded five times in random order. Two of these sequences were produced, for a total of 80 triad presentations. As in Experiment 1, each triad was $6 \mathrm{sec}$ long, and an ISI of $4 \mathrm{sec}$ was placed between them. The sequences were also presented to the subjects with the video monitor switched off, serving as an additional control.

The presentation tape also included audiovisual identifications, audio-alone identifications, and video-alone identifications. These three conditions were arranged in the same way as described in Experiment 1 . All conditions were counterbalanced for presentation to the subjects.

Subjects. Twenty-four undergraduates at the University of California, Riverside, participated for partial fulfillment of a class requirement. All subjects reported having normal or corrected-tonormal vision and normal hearing. None of the subjects had participated in Experiment 1.

Procedure. Six subjects were presented with the conditions in the order: audio-alone $A X B$, audiovisual $A X B$, audio-alone $A X B$, audiovisual $A X B$, audiovisual identification, video identification, and audio identification. Eight subjects were presented with the conditions in the order: audiovisual identification, audio-alone $A \times B$, audiovisual $A X B$, audio-alone identification, audiovisual $A X B$, video identification, and audio identification. Seven subjects were presented with the conditions in the order: audiovisual identification, audiovisual $A X B$, audiovisual $A X B$, audio-alone $A X B$, audioalone $A X B$, video identification, and audio identification. The re- 
Table 2

Percentage of Correct Responses per Subject for Audiovisual Identification, Audiovisual Discrimination, and Audio-Alone Discrimination for Experiment 2

\begin{tabular}{cccc}
\hline Subject & Audiovisual ID & Audiovisual AXB & Audio-Alone AXB \\
\hline 1 & 10 & 85 & 85 \\
2 & 0 & 80 & 100 \\
3 & 40 & 100 & 100 \\
4 & 10 & 100 & 70 \\
5 & 10 & 75 & 100 \\
6 & 30 & 100 & 100 \\
7 & 20 & 60 & 100 \\
8 & 20 & 75 & 95 \\
9 & 0 & 55 & 100 \\
10 & 0 & 85 & 90 \\
11 & 0 & 85 & 100 \\
12 & 90 & 70 & 100 \\
13 & 40 & 100 & 95 \\
14 & 0 & 65 & 100 \\
15 & 10 & 35 & 65 \\
16 & 20 & 60 & 75 \\
17 & 10 & 85 & 95 \\
18 & 10 & 75 & 100 \\
19 & 0 & 100 & 100 \\
20 & 0 & 70 & 100 \\
21 & 90 & 80 & 100 \\
22 & 40 & 100 & 100 \\
23 & 20 & 70 & 100 \\
24 & 30 & 50 & 100 \\
$M$ & 20.8 & 77.5 & 95.2 \\
SD & 25.2 & 17.8 & 9.6 \\
\hline & & &
\end{tabular}

maining 3 subjects received the conditions in the order: audiovisual $\mathrm{AXB}$, audio-alone $\mathrm{AXB}$, audiovisual $\mathrm{AXB}$, audio-alone $\mathrm{AXB}$, audiovisual identification, video identification, and audio identification.

The instructions for Experiment 2 were the same as those given in Experiment 1. The subjects were shown two examples of the matching task before the experiment began. These examples were audiovisual control triads so the subjects would be able to match the $\mathrm{X}$ token to one of the A or B tokens. Again, the subjects were told that it was critical that they look back up at the video monitor as soon as they completed each judgment. The experimenter watched the subjects to ensure that they did see all of the presentations. The experiment lasted approximately $1 \mathrm{~h}$.

\section{Results and Discussion}

Analysis of all subjects' data. Pooled results for certain critical conditions of Experiment 2 are shown in Figure 2A.

The percentage of each subject's correct responses to the discrepant audiovisual identification trials can be seen in column 2 of Table 2 . The mean percentage of correct responses across subjects was $21 \%$ (see Figure $2 \mathrm{~A}$ ). This outcome means that the subjects heard the audio $/ \mathrm{ba} /$-visual $/ \mathrm{va} /$ as the visually influenced $/ \mathrm{va} / 79 \%$ of the time. It is not clear why there seems to be less of a visual influence here than in Experiment 1, which used the same stimuli and elicited $96 \% / \mathrm{va} /$ responses. However, as can be seen from Table 2 , a few subjects did not demonstrate McGurk effects. The percentage correct for the consistent /ba/ audiovisual identification was $98 \%$, and the percentage correct for the consistent audiovisual / $\mathrm{va} /$ token was $100 \%$. The percentages correct for the audio identification trials were $100 \%$ for $/ \mathrm{va} /$ and $95 \%$ for $/ \mathrm{ba} /$.
Finally, the mean percentages for the video-alone identifications were also calculated for this experiment. For the $/ \mathrm{ba} /$ video token, there were $97 \%$ correct $/ \mathrm{ba} /$ responses, $2 \% / \mathrm{va} /$ responses, and $1 \% / \mathrm{fa} /$ responses. For the $/ \mathrm{va} /$ token, there were $89 \%$ correct $/ \mathrm{va} /$ responses and $11 \% / \mathrm{ba} /$ responses. Finally, for the $/ \mathrm{fa} /$ video token, there were $0 \%$ correct $/ \mathrm{fa} /$ responses, $98 \% / \mathrm{va} /$ responses, and $2 \% / \mathrm{ba} /$ responses. Thus, again, while the $/ \mathrm{ba} /$ and / va/ tokens generally looked to the subjects as they were supposed to, the /fa/ visual token was identified mostly as $/ \mathrm{va} /$.

The percentages of correct responses for the critical audiovisual AXB triads for each subject are shown in column 3 of Table 2 . The pooled mean of correct responses was $78 \%$, which is significantly greater than chance $[t(23)=7.562, p<.05]$. This result means that overall, the subjects more often chose the audio / va/ to match the compatible audio / va/-video / fa/ than to match the discrepant audio /ba/-video / fa/. There was no effect of stimulus presentation order on responses for the critical audiovisual AXB triads $[F(3,20)=.428, p>.05]$. The percentages of correct responses for the audio-alone AXB triads are shown in column 4 of Table 2 . The pooled percentage correct for this condition was found to be $95 \%$. The percentage correct for the audiovisual control triads was $95 \%$.

Using $t$ tests to compare across conditions, it was found that the percentage correct for the critical audiovisual AXB triads $(78 \%)$ was significantly less than the percentage correct for the analogous audio-alone AXB triads (95\%) $[t(23)=-4.633, p<.05]$, suggesting that there was 
a significant video influence for the discrimination condition.

Analysis of data from subjects who show McGurk effect. A test of phonetic equivalence between a visually influenced and a consistent $/ \mathrm{va} /$ would require that the subjects identify both the syllables as $/ \mathrm{va} /$ most of the time. As can be seen in Table 2, this is not the case for some of the subjects in Experiment 2. It would seem that a more appropriate comparison of the visually influenced and the audiovisually compatible / $\mathrm{va}$ / in a matching task would be based on data from the subjects who identified the visually influenced token as $/ \mathrm{va} /$ at least $90 \%$ of the time. A subsequent analysis was conducted on the 13 subjects who reached this (somewhat arbitrary) criterion. Pooled results of certain critical conditions for these subjects are shown in Figure 2B.

For these subjects, the mean percentage of correct responses for the incompatible identification token was $5 \%$. Thus, on average, these subjects reported hearing this token as $/ \mathrm{va} /$ for $95 \%$ of the trials. Their mean percentage correct scores for the consistent $/ \mathrm{ba} /$ token and $/ \mathrm{va} /$ token were $99 \%$ and $100 \%$, respectively. Their percentages correct for the audio identification trials were $100 \%$ for the $/ \mathrm{va} /$ and $93 \%$ for the $/ \mathrm{ba} /$. For the video identification, the percentage correct for the / fa/ token remained the same as in the previous analysis, the percentage correct for the $/ \mathrm{ba} /$ was $100 \%$, and the percentage correct for the $/ v a /$ was $87 \%$.

The percentage of correct responses for the critical AXB triads for these selected subjects was $77 \%$, only $1 \%$ lower than the percentage for all subjects. Again, this result was significantly greater than chance $[t(12)=5.399$, $p<.05]$. Thus, these "McGurk-selected" subjects also preferred to match the audio alone / $\mathrm{va} /$ to the audiovisually compatible $/ \mathrm{va} /$. The percentage correct for the audioalone AXB triads was $93 \%$, which was significantly different from the percentage correct for the critical audiovisual triads $[t(12)=-3.032, p<.05)$, indicating that there was a significant influence of video on these selected subjects. Finally, the percentage correct for the audiovisual control triads was $96 \%$. Thus, it seems that even the subjects who reported hearing the audiovisually incompatible tokens as /va/ at least $90 \%$ of the time still preferred to match the audio-alone token to the audiovisually compatible token when asked to perform a discrimination task.

Why might subjects prefer an audiovisually compatible match? At least two alternative explanations come to mind, both of which assume that the subjects continue to hear the visually influenced token as /va/ in the AXB context. First, it could be that although the subjects hear the visually influenced token as a /va/, they hear it as less phonetically convincing than the audiovisually compatible token. If so, they would prefer the audiovisually compatible token to match with the audio-alone /va/. On the other hand, the subjects might be making matches on the basis of similarities in phonetically extraneous properties. "Phonetically extraneous acoustic properties" describes properties that are not relevant to the phonetic classifica- tions at hand. For example, even though syllable duration might be relevant for phonetic classification in some contexts, it would most likely not be relevant in influencing classification of the $/ \mathrm{ba} /$ and $/ \mathrm{va} /$ tokens used here.

Thus, the subjects might still hear the visually influenced token as a phonetically convincing / va/. However, the subjects might hear the visually influenced $/ \mathrm{va} /$ to have less in common in phonetically extraneous properties with the audio-alone / va/ than does the consistent audiovisual $/ \mathrm{va} /$ token. This second hypothesis seems compatible with the identification results reported here and elsewhere, which imply that visually influenced syllables can be phonetically compelling. In addition, a similar argument has been made to explain why there is some degree of discrimination between (auditory) speech tokens within a phonetic category (Borden \& Harris, 1984; Liberman et al., 1957).

In Experiment 3, we address this hypothesis by manipulating acoustic characteristics of audio tokens that should be extraneous to phonetic strength.

\section{EXPERIMENT 3}

In Experiment 2, the critical discrimination triads involved tokens that were generally similar in intonation, timbre, and duration. This is because the $/ \mathrm{ba} /$ used was generated by modifying the $/ \mathrm{va}$ / token. Although the general acoustic characteristics were the same across all three tokens in a given triad, the two /va/ tokens were exactly alike acoustically, differing from the $/ \mathrm{ba} /$ in onset and duration. Thus, the two / va/ tokens in each triad had more in common in two different ways: (1) both shared acoustic properties that were relevant to phonetic identifications (e.g., degree of frication, formant structure), and (2) both shared properties that were extraneous to phonetic identifications (e.g., duration, absolute onset intensity). ${ }^{1}$ Thus, in interpreting the results of the critical discrimination condition, it is not possible to determine whether the subjects' matching of the two /va/s was based on phonetically relevant or extraneous similarities.

Experiment 3 was designed to determine whether the observed matching judgments were based on relevant or extraneous phonetic properties. Because we were unsure as to what the salient extraneous properties could have been in Experiment 2, we decided to manipulate an extraneous dimension that we knew would be obvious to the subjects. Accordingly, synthetic tokens were generated that differed significantly from each other in absolute fun-

Table 3

Values of Fundamental Frequency of Five Tokens Used in Experiment 3

\begin{tabular}{rrrrrr}
\hline Time & $\mathrm{Ba}^{*}$ & $\mathrm{Ba}^{-}$ & $\mathrm{Va}$ & $\mathrm{Va}^{+}$ & $\mathrm{Va}^{-}$ \\
\hline 0 & 100 & 90 & 95 & 100 & 90 \\
40 & 110 & 100 & 105 & 110 & 100 \\
120 & 105 & 95 & 100 & 105 & 95 \\
220 & 100 & 90 & 95 & 100 & 90 \\
420 & 85 & 75 & 80 & 85 & 75 \\
600 & 65 & 55 & 60 & 65 & 55 \\
\hline
\end{tabular}

*In milliseconds. 
damental frequency (F0) contour. (The tokens were generated with the same relative contours but differed from each other in absolute F0 from moment to moment; see Table 3.) The primary perceptual difference for such tokens was a clearly noticeable difference in pitch. The critical triads in Experiment 3 involved an audio $/ \mathrm{ba} /$-video $/ \mathrm{fa} /$ and an audio /va/-video / fa/ in either the $A$ or $B$ position. The $X$ token again consisted of an audio-alone /va/. The F0s for the audio components of the tokens were all different from each other. The F0 of the $\mathrm{X}$ token was halfway between the fundamental frequencies of the $A$ and $\mathrm{B}$ tokens so that the $\mathrm{A}$ and $\mathrm{B}$ tokens differed from the $\mathrm{X}$ token by the same frequency increment (but not necessarily the same pitch increment). It was thought that manipulating the surface acoustic characteristics (F0) in this way might force the subjects to base judgments on phonetically relevant properties rather than on properties extraneous to phonetic categorization. ${ }^{2}$

Given this experimental design, the hypotheses are as follows: If, in Experiment 2, the subjects' matches were based on phonetically relevant properties, then the F0 manipulation should not affect the patterning of results. The subjects should still prefer to match the audio alone /va/ to the audiovisually compatible / $\mathrm{va}$. If, however, the subjects based their matches on some phonetically extraneous similarity, then this matching preference might be nullified by the significant F0 changes in the current stimuli. Hence, the subjects would be forced to base their judgments on phonetically relevant properties. Since both the compatible and incompatible tokens would sound like $/ \mathrm{va} / \mathrm{s}$, it would be predicted that the subjects would choose the two audiovisual tokens equally often.

This hypothesis will only be supported if the subjects choose the visually influenced and compatible tokens equally often, regardless of which fundamental frequency each token has been assigned. Although F0s are used that are equidistant from the middle (X) token's F0, the subjects could still perceive either the higher or lower F0 as being more similar to the middle token's F0. For example, consider a situation in which a subject perceived the lower F0 as closer in pitch to the middle F0. This subject might then equally match an $X$ token to (1) an $A$ token that was audiovisually discrepant with a lower F0 audio component and (2) a B token that was audiovisually compatible with a higher $\mathrm{F} 0$ audio component. This outcome could be given two interpretations. First, the subject could be basing the matches on purely phonetically relevant properties, thereby hearing both $\mathrm{A}$ and $\mathrm{B}$ syllables as equally compelling $/ \mathrm{va} / \mathrm{s}$. However, the subject might be basing the match on both phonetically relevant and extraneous (F0) properties. In this case, the subject would hear the compatible token as more convincing phonetically, but not so much more as to override the perceived pitch difference heard to be greater for the compatible token. Thus, a control is needed to ensure that if the subjects choose tokens equally often, they do so on the basis of a phonetic similarity. Accordingly, two critical triad conditions were implemented that swap the fundamental frequency for the discrepant and compatible tokens.

\section{Method}

Stimuli. The audio stimuli were generated using a Klatt 80 Cascade/Parallel Speech Synthesizer (Klatt, 1980), which was operated from a COMPAQ 386/25 computer. The synthesis values were generally based on parameters outlined by Klatt. Two /ba/ syllables and three /va/ syllables were generated. All syllables were $600 \mathrm{msec}$ in duration, which matched the natural tokens used in Experiments 1 and 2 and was most compatible with the visual displays. Three formants were manipulated to produce each syllable. Other formants were set to default values. All tokens shared the same center formant frequencies during the steady-state (vocalic) portions of the syllables. These formant values were centered at 700,1220 , and $2600 \mathrm{~Hz}$ for the first (F1), second (F2), and third (F3) formants, respectively.

Each $/ \mathrm{ba} /$ syllable contained a 15 -msec initial formant transition followed by a 585-msec steady-state vowel. The onset frequencies of the first, second, and third formant transitions were 200, 1100 , and $2050 \mathrm{~Hz}$, respectively. The formant bandwidths started at 60 , 110 , and $130 \mathrm{~Hz}$ for $F 1, F 2$, and $F 3$, respectively. These bandwidths changed toward and were then maintained at 130,70 , and $160 \mathrm{~Hz}$ (for F1, F2, and F3) by the steady-state portions of the syllables. The $/ \mathrm{va}$ / tokens contained an 85 -msec initial formant transition, followed by a 515 -msec steady-state vowel. The onset frequencies of the first, second, and third formant transitions for these tokens were 220,1100 , and $2080 \mathrm{~Hz}$. The / va/formant bandwidths started at 60,90 , and $120 \mathrm{~Hz}$ for $F 1, F 2$, and F3, respectively. These bandwidths changed toward and were then maintained at 130 , 70 , and $160 \mathrm{~Hz}$ (for F1, F2, and F3) by the steady-state portions of the syllables.

These tokens were synthesized with various F0 contours. One of the $/ \mathrm{ba} /$ tokens $\left(/ \mathrm{ba} /{ }^{+}\right)$had a fundamental frequency that was higher overall relative to the middle (X position)/va/ token, and the other $\mathrm{ha} /$ token $\left(/ \mathrm{ba} /{ }^{-}\right)$had a fundamental frequency that was lower overall than the middle / $/ \mathrm{va} /$ token. The two remaining $/ \mathrm{va} /$ tokens had fundamental frequencies that were analogous to the two /ba/ tokens (hence $/ \mathrm{va} /{ }^{+}$and $/ \mathrm{va}^{-}$). The F0 contours of these tokens are reported in Table 3. These audio tokens were dubbed onto video tokens of /ba/ and $/ \mathrm{fa} /$ in the manner described previously.

The AXB triads were arranged in the following manner: The $\mathrm{X}$ token was always the audio-alone / $v a /$ token, which differed from all other tokens in F0. The A and B tokens in the triads were always audiovisual and always different from each other. For the four critical triads, the A and B tokens were a discrepant token (audio /ba/-video/fa/) and a consistent token (audio/va/-video/fa/). These $A$ and $B$ tokens always differed from each other and the $X$ token in F0. Two of the triads included A and B tokens with $/ \mathrm{ba}^{+}$and $/ \mathrm{va} /{ }^{-}$audio components. An example of this type of triad is shown schematically in Figure 3A. The other two triads involved $/ \mathrm{ba} /{ }^{-}$ and $/ \mathrm{va} /{ }^{+}$components, an example of this type of triad is shown schematically in Figure 3B. As stated previously, this manipulation was important in ensuring that if subjects chose both discrepant and consistent tokens equally often, they did so on the basis of phonetic equality and not on the basis of tradeoff between phonetic and F0 characteristics. The positions of the A and B tokens were switched for both of these triad pairs, producing four critical triads altogether.

Four control triads were also created. For these triads, the $A$ and B tokens were both consistent audiovisually (audio $/ \mathrm{ba} /$-video $/ \mathrm{ba} /$, audio /va/-video /fa/). The F0 values for the tokens in the control triads were analogous to the critical tokens.

The triads lasted $6 \mathrm{sec}$ and had an ISI of $4 \mathrm{sec}$. The 8 triads were recorded 10 times each in random order. One block of $\mathbf{4 0}$ triads 
(A)

\begin{tabular}{|c|c|c|}
\hline & $\underline{\mathrm{A}}$ & $\underline{X}$ \\
\hline Audio & /ba/t+ & $/ \mathrm{va} /$ \\
\hline Video & $/ \mathrm{fa} /$ & \\
\hline Mean \% Matches & 33 & \\
\hline $\begin{array}{l}\text { Mean \% Correct Identifications } \\
\text { (based on audio portion } \\
\text { of simulus) }\end{array}$ & 2 & \\
\hline
\end{tabular}

$\begin{array}{llll} & \underline{\mathrm{A}} & \underline{\mathrm{X}} & \underline{\mathrm{B}} \\ \text { Audio } & / \mathrm{ba} /- & / \mathrm{va} / & / \mathrm{va} /+ \\ \text { Video } & / \mathrm{fa} / & & / \mathrm{fa} /\end{array}$

Mean \% Matches

Mean \% Correct Identifications

(based on audio portion

of stimulus)
39

3
61

92

Figure 3. Two examples of critical AXB matching conditions used in Experiment 3 and mean percentage results of critical conditions (see text for details).

was presented to the subjects with the video monitor switched off ( 8 triads $\times 5$ repetitions). This condition served as an additional control to determine if the subjects would normally match the two iva/ tokens.

The presentation tape also included audio and audiovisual identification stimuli. The audio identification stimuli consisted of the 5 synthesized tokens. Each token was presented 5 times in random order, with an ISI of $3 \mathrm{sec}$. The stimuli for the audiovisual identification consisted of the $2 / \mathrm{ba} /$ tokens $\left(/ \mathrm{ba} /{ }^{+}\right.$and $\left./ \mathrm{ba} /{ }^{-}\right)$along with the 2 analogous $/ \mathrm{va} /$ tokens $\left(/ \mathrm{va}^{+}\right.$and $/ \mathrm{va} /{ }^{-}$), each paired with $\mathrm{a} / \mathrm{fa} / \mathrm{video}$. In addition, the stimuli for this condition included the two $/ \mathrm{ba} /$ tokens paired with a / ba/ video. The consistent $/ \mathrm{ba} /$ tokens were presented 20 times each, and the remaining 4 tokens were presented 10 times each, for a total of 80 presentations. These tokens were also recorded in random order, with an ISI of $3 \mathrm{sec}$.

Subjects. Thirteen undergraduates at the University of California, Riverside, participated in the experiment for partial course credit. All subjects reported having normal or corrected-to-normal vision and normal hearing. None of the subjects had participated in Experiment 1 or 2.

Procedure. The subjects were run in groups of 2 or 3 . Six subjects received the conditions in the order: audiovisual $\mathbf{A X B}$, audioalone AXB, audio identification, and audiovisual identification. Three subjects received the conditions in the order: audio-alone $A X B$, audiovisual $A X B$, audio identification, and audiovisual identification. Four subjects received conditions in the order: audio identification, audiovisual identification, audiovisual $\mathrm{AXB}$, and audio-alone $\mathrm{AXB}$. The general instructions for Experiment 3 were the same as those given in Experiments 1 and 2.

Data collection, however, was slightly different for this experiment. The subjects were seated in front of a Macintosh Plus com- puter. For the matching trials, the computer screen showed the letters $A, X$, and $B$. As in the other experiments, the subjects were instructed to choose the $A$ if the middle token sounded more like the first token or the $B$ if the middle token sounded more like the third token. For this experiment, they chose A or B by moving a computer mouse to position a cursor on the letter. The subjects were allowed to change their answers if they made errors in responding.

In the identification condition, the subjects were asked to specify the syllable that they heard by typing in its first letter on the computer keyboard. The specified syllable was then displayed on the screen. As in the matching task, the subjects were allowed to change their answers.

The subjects were presented two examples of the matching task before the experiment actually began. These examples were audiovisual control triads, allowing the subjects to match the $X$ token to one of the A or B tokens. The Macintosh computers were separated by $3 \mathrm{ft}$ and angled so that each subject could only see the computer monitor he/she was responding on. Again, the subjects were told that it was critical that they look back up at the video screen as soon as they completed each judgment and were monitored accordingly. The experiment lasted approximately $1 \mathrm{~h}$ for each subject.

\section{Results and Discussion}

Pooled results for certain critical conditions are shown in Figure 3A for the triads involving the $/ \mathrm{ba} /{ }^{+}$token and Figure 3B for the triads involving the $/ \mathrm{ba}^{-}$token.

The mean percentage for each subject's identification responses to the discrepant audiovisual trials can be seen in columns 2 and 3 of Table 4 . The pooled mean percent- 
Table 4

Percentage of Correct Responses per Subject for Audiovisual Identification, Audiovisual Discrimination, and Audio-Alone Discrimination for Experiment 3

\begin{tabular}{|c|c|c|c|c|c|c|}
\hline Subject & $\begin{array}{c}\text { Audiovisual } \\
\text { ID } / \mathrm{ba} /{ }^{+}\end{array}$ & $\begin{array}{l}\text { Audiovisual } \\
\mathrm{ID} / \mathrm{ba}^{-}\end{array}$ & $\begin{array}{c}\text { Audiovisual } \\
\text { "/ba/ }{ }^{+}, \text {AXB }\end{array}$ & $\begin{array}{c}\text { Audiovisual } \\
\text { " } / \mathrm{ba} /^{-}, " \text { AXB }\end{array}$ & $\begin{array}{l}\text { Audio-Alone } \\
\text { " } / \mathrm{ba} /{ }^{+} " \text { AXB }\end{array}$ & $\begin{array}{l}\text { Audio-Alone } \\
\text { " } \mathrm{ba}{ }^{-"} \text { AXB }\end{array}$ \\
\hline 1 & 0 & 10 & 75 & 65 & 100 & 90 \\
\hline 2 & 0 & 0 & 40 & 65 & 100 & 100 \\
\hline 3 & 10 & 0 & 65 & 50 & 100 & 100 \\
\hline 4 & 0 & 0 & 55 & 70 & 84 & 100 \\
\hline 5 & 0 & 10 & 75 & 85 & 95 & 100 \\
\hline 6 & 0 & 0 & 95 & 90 & 100 & 95 \\
\hline 7 & 0 & 0 & 95 & 45 & 100 & 100 \\
\hline 8 & 0 & 10 & 60 & 50 & 100 & 90 \\
\hline 9 & 0 & 0 & 85 & 70 & 100 & 100 \\
\hline 10 & 0 & 0 & 70 & 45 & 84 & 80 \\
\hline 11 & 0 & 0 & 65 & 60 & 100 & 100 \\
\hline 12 & 10 & 10 & 55 & 55 & 100 & 100 \\
\hline 13 & 0 & 0 & 40 & 40 & 100 & 100 \\
\hline$M$ & 1.5 & 3.1 & 60.8 & 67.3 & 97.2 & 96.5 \\
\hline$S D$ & 1.0 & 4.8 & 15.4 & 17.9 & 6.0 & 6.3 \\
\hline
\end{tabular}

ages of correct responses for the discrepant audiovisual identification trials were $2 \%$ for the $/ \mathrm{ba} /{ }^{+}$token and $3 \%$ for the $/ \mathrm{ba} /{ }^{-}$token. Thus, the subjects heard the audio $/ \mathrm{ba} /$-video $/ \mathrm{fa} /$ tokens as visually influenced / va/tokens $97 \%$ and $98 \%$ of the time. The fact that both tokens, which differed in relative F0, showed a strong effect suggests that in this context, the F0 manipulation was, indeed, phonetically extraneous. The percentage correct scores for the consistent audiovisual $/ \mathrm{ba} /{ }^{+}$and $/ \mathrm{ba} /{ }^{-}$ tokens were both $99 \%$. The percentage correct scores for the consistent audiovisual $/ \mathrm{va} /{ }^{+}$and $/ \mathrm{va} /{ }^{-}$tokens were $92 \%$ and $97 \%$, respectively. The low mean percentage correct for the audiovisual $/ \mathrm{va} /{ }^{+}$token was primarily due to 1 subject, who reported hearing $/ \mathrm{ra} / 100 \%$ of the time. The mean percentage correct for the audio identification trials was $95 \%$ and $97 \%$ for the $/ \mathrm{ba} /{ }^{+}$and $/ \mathrm{ba} /{ }^{-}$tokens and $94 \%$ and $92 \%$ for the $/ \mathrm{va} /{ }^{+}$and $/ \mathrm{va} /{ }^{-}$tokens. The mean percentage correct for the middle / va/ token was $92 \%$. Again, the lower percentage of correct $/ \mathrm{va} / \mathrm{re}-$ sponses was primarily due to 1 subject, who reported hearing $/ \mathrm{ra} /$ for many of the trials.

The percentage of correct responses for the critical audiovisual AXB triads for each subject is shown in columns 4 and 5 of Table 4 . The pooled mean percentage correct for the critical AXB triads was $67 \%$ for the triads including the $/ \mathrm{ba} /{ }^{+}$token and $61 \%$ for the triads including the $/ \mathrm{ba} /{ }^{-}$token. Both of these scores are significantly greater than chance $[t(12)=3.49, p<.05$, for the $/ \mathrm{ba} /{ }^{+}$triad and $t(12)=2.52, p<.05$, for the $/ \mathrm{ba} /{ }^{-}$triad]. Thus, overall, the subjects still preferred to match the middle audio / $\mathrm{va} /$ token to the consistent audiovisual /va/token, regardless of F0. There was no presentation ordering effect for either critical AXB triad $\left[F(2,10)=3.506, p>.05\right.$, for $/ \mathrm{ba} /{ }^{+}$triads and $F(2,10)=1.465, p>.05$, for $/ \mathrm{ba} /{ }^{-}$triads]. The percentages of correct responses for the audio-alone triads are shown in columns 6 and 7 of Table 4 . The pooled percentage correct for the audio-alone AXB trials was $97 \%$ for both types of triads.
Using $t$ tests to compare across conditions, it was found that the percentages correct for both of the audiovisual AXB conditions (67\% and $61 \%$ ) were significantly less than the percentages correct for the analogous audio-alone AXB triads (97\% and 97\%) $[t(12)=-5.87, p<.01$, for the former test and $t(12)=-8.38, p<.01$, for the latter], suggesting that, again, there was a significant video influence for the critical discrimination conditions.

To determine if the F0 manipulation had any significant influence on matching preferences, a comparison was conducted between correct responses for the critical AXB condition in Experiment 2 and the two critical AXB conditions in Experiment 3. An analysis of variance revealed that there was no significant difference among the three conditions $[F(2,24)=2.74, p>.05]$.

Thus, the subjects matched the audio-alone /va/ to a consistent audio /va/-video /fa/ significantly more often than to a discrepant audio /ba/-video / fa/, regardless of an extraneous acoustic manipulation (F0). This outcome might indicate that the subjects' preferences are, in fact, based on phonetically relevant and not extraneous similarities in the stimuli. This conclusion would imply that the visually influenced stimulus is not phonetically equivalent to the consistent audiovisual stimulus.

At least one other interpretation does exist, however. It could be that subjects do not base their matching judgments on either phonetically relevant or extraneous similarity. Instead, subjects might base their preferences on the relative audiovisual discrepancy of the consistent and discrepant tokens. In other words, faced with three tokens that all sound the same (in terms of phonetically relevant and extraneous properties), subjects might resort to the crossmodal appearance of the tokens and choose the more congruent, audiovisually compatible token for the match.

There is some evidence that observers can be aware of these discrepancies, yet still report hearing the visually influenced syllable. For example, Repp et al. (1983) had subjects rate audiovisual compatibility as well as identify a series of dubbed audiovisual $\mathrm{C} / \mathrm{a} /$ syllables. They found 
that compatibility ratings depended on the distance between acoustically and optically specified place of articulation for the consonant. Interestingly, the subjects' relative awareness of incompatibility had little bearing on the degree of visual influence on the heard syllables.

Summerfield and McGrath (1984) have reported similar findings for audiovisual vowels. They also found that subjects were accurate at determining the audiovisual incompatibility of vowels embedded in a $/ \mathrm{b} / \mathrm{V}$ context. Even though they initially observed that the degree of incompatibility perceived was correlated with the strength of the visual influence, a subsequent experiment suggested that visual information does influence "heard" vowels even when subjects detect audiovisual discrepancies.

Thus, the subjects can notice a discrepancy and still display the McGurk effect. Given this observation, it could be that in both Experiments 2 and 3 the subjects heard the consistent and discrepant tokens as phonetically equal /va/s and based their matching judgments simply on relative audiovisual discrepancy. Clearly, in order for this explanation to be true of our stimuli, the audiovisually discrepant tokens used in Experiments 2 and 3 would have to be noticeably more discrepant than their audiovisually compatible counterparts. This issue was tested in Experiment 4 .

\section{EXPERIMENT 4}

The hypothesis for Experiment 4 is as follows: If the subjects based their matching judgments on noticeable relative discrepancies in Experiments 2 and 3, then the audio $/ \mathrm{ba} /$-video $/ \mathrm{fa} /$ tokens should be rated as more discrepant than the audio /va/-video /fa/tokens.

\section{Method}

Stimuli. The stimuli used for this experiment were the same natural audiovisual identification tokens used in Experiment 2 and the same synthetic audiovisual identification tokens used in Experiment 3.

Subjects. Twelve subjects judged the natural stimuli, and 13 subjects judged the synthetic stimuli. The subjects were undergraduates at the University of California, Riverside, and participated for partial fulfillment of a class requirement. All subjects reported having normal or corrected-to-normal vision and normal hearing. None of the subjects had participated in any of the first three experiments.

Procedure. The subjects were run in groups of 2 or 3 . The subjects were instructed to watch as well as listen to the presented audiovisual tokens. They were then to write down the first letter of the syllable that they heard. In addition, the subjects were asked to rate whether they felt the audiovisual tokens had been dubbed or not on a 6-point scale ( 1 = dubbed; 6 = not dubbed; Repp et al., 1983). The same monitoring procedures used for Experiments 1-3 were used here to ensure that the subjects (1) saw each full presentation and (2) did not base their judgments on a neighboring subject's responses.

\section{Results and Discussion}

The mean percentage of correct identification responses for the natural audiovisually discrepant token was $11 \%$, indicating that the subjects reported hearing the token as $/ \mathrm{va} /$ on $89 \%$ of the presentations. The mean percentage correct for the consistent / va/ tokens was $98 \%$. The discrepancy ratings for the natural tokens were 4.64 for the audiovisually compatible token and 4.34 for the audiovisually discrepant token. A $t$ test revealed that this was not a significant difference in discrepancy ratings for these tokens $[t(11)=.865, p>.05]$.

The mean percentages of correct identification responses for the synthetic audiovisually discrepant tokens were $5 \%$ for the $/ \mathrm{ba} /{ }^{+}$token and $2 \%$ for the $/ \mathrm{ba} /{ }^{-}$token. This meant that the subjects reported hearing these tokens as $/ \mathrm{va} / 95 \%$ and $98 \%$ of the time. The mean percentages of correct responses for the audiovisually consistent $/ \mathrm{va} /{ }^{+}$ and $/ \mathrm{va} /{ }^{-}$tokens were $99 \%$ and $98 \%$, respectively. The mean percentage correct for both consistent $/ \mathrm{ba} /$ tokens was $100 \%$.

The discrepancy ratings of interest were the ratings for the audiovisually discrepant tokens and the consistent / va/ tokens, since these were the tokens used in the critical AXB triads. The discrepancy rating pooled across subjects for the discrepant $/ \mathrm{ba} /{ }^{-}$token was 4.42 . The pooled discrepancy rating for the discrepant $/ \mathrm{ba} /{ }^{+}$token was 4.14. The discrepancy ratings for the consistent $/ \mathrm{va} /{ }^{+}$and $/ \mathrm{va} /{ }^{-}$tokens were 4.57 and 4.69 , respectively. We used $t$ tests to compare the discrepancy ratings of the $/ \mathrm{ba} /{ }^{-}$ and $/ \mathrm{va} /{ }^{+}$tokens and, subsequently, the $/ \mathrm{ba} /{ }^{+}$and $/ \mathrm{va} /{ }^{-}$ tokens, since these were the comparisons made in the AXB triads of Experiment 3. There was no significant difference between the $/ \mathrm{ba} /{ }^{-}$and $/ \mathrm{va} /{ }^{+}$tokens $[t(12)=$ $-1.212, p>.05$ ]; however, there was a significant difference between the discrepancy ratings for the $/ \mathrm{ba} /{ }^{+}$and $/ \mathrm{va} /{ }^{-}$tokens $[t(12)=-2.71, p<.05]$.

This outcome means that the subjects could potentially base their $/ \mathrm{ba} /{ }^{+}-/ \mathrm{va} /{ }^{-}$triad, but not their $/ \mathrm{ba} /{ }^{-}-/ \mathrm{va} /{ }^{+}$ triad, matches on relative discrepancy. However, in order for this scenario to be meaningful, it would have to be reflected in the AXB findings of Experiment 3. In other words, if the subjects were using this relatively greater $/ \mathrm{ba} /{ }^{+}$discrepancy to help them make their matching judgments, we would expect that the subjects would be better at matching for the $/ \mathrm{ba} /{ }^{+}-/ \mathrm{va} /{ }^{-}$triad than for the $/ \mathrm{ba} /{ }^{-}-/ \mathrm{va} /{ }^{+}$triad. A $t$ test revealed that there was not a significant difference for the percentage of correct responses between these two conditions $(67 \%$ correct for the $/ \mathrm{ba} /{ }^{+}-/ \mathrm{va} /{ }^{-}$triad vs. $61 \%$ correct for the $/ \mathrm{ba} /{ }^{-}-/ \mathrm{va} /{ }^{+}$triad) $[t(12)=-1.257, p>.05]$. Therefore, it seems that although the subjects did report the $/ \mathrm{ba} /{ }^{+}$token as being more discrepant, it was not the general basis for matching preferences.

Overall, the results of Experiment 4 indicated that the subjects generally did not notice discrepancy differences between discrepant and compatible tokens. This outcome suggests that matching preferences in Experiments 2 and 3 were not based on noticeable relative audiovisual discrepancies.

It could be, however, that discrepancies that went unnoticed influenced matching judgments. For example, Green and Kuhl (1991) observed that although subjects failed to notice discrepancies in various audiovisual tokens, their reaction time in labeling the tokens depended 
on the relative discrepancy. Thus, subjects reported hearing no discrepancy even though discrepancy seemed to slow down their identification judgments. The same unnoticed discrepancy could influence the matching judgments observed here. Future research will be designed to address this question.

\section{GENERAL DISCUSSION}

The overall goal of these experiments was to determine if a visually influenced syllable is phonetically equivalent to its audiovisually compatible counterpart. In addition, Experiment 1 was designed to test a compelling visually influenced syllable in an AXB matching context. Experiment 1 demonstrated that subjects identify a token made up of audio $/ \mathrm{ba} /-\mathrm{visual} / \mathrm{fa} / \mathrm{as} / \mathrm{va} /$ and match it to an audio-alone $/ \mathrm{va} /$ significantly more often than to an audioalone $/ \mathrm{ba} /$. Although subjects match the visually influenced $/ \mathrm{va} /$ to an audio $/ \mathrm{va} /$ less often than they identify it as a $/ \mathrm{va} /$, the results suggest that the visually influenced /va/ was still convincing under the more stringent test of AXB matching.

The remaining experiments tested the issue of phonetic equivalence using an AXB paradigm. Given identification results reported here and elsewhere, it was predicted that subjects would hear both compatible and visually influenced presentations of the AXB stimuli as phonetically equivalent. Accordingly, subjects should then match the two audiovisual presentations to an audio-alone / va/ equally often. Contrary to this prediction, however, Experiments 2 and 3 demonstrated that subjects are more likely to match an audio / va/ to an audiovisually compatible /va/ than to a visually influenced /va/. In addition, Experiment 3 also indicated that this preference might not be based on a phonetically extraneous acoustic property (F0) of the tokens. Finally, Experiment 4 suggested that the preference for the compatible syllable match was not based on noticeable relative discrepancy.

Taken together, these results might suggest that even a compelling visually influenced syllable is not phonetically equivalent to its audiovisually compatible counterpart. This interpretation could imply that the McGurk effect is not as strong as results of previous identification studies have suggested (e.g., Green et al., 1991; Repp et al., 1983). Recent evidence based on Japanese observers (and using identification judgments) also suggests that the McGurk effect does have limits (Sekiyama \& Tohkura, 1991).

Although our results suggest that the visually influenced and audiovisually compatible syllables are not phonetically equivalent, this does not rule out the possibility that they are equally perceptually convincing. For example, it has been assumed that our visually influenced syllable generally has been heard as a / va/ since subjects label it as such. However, it is possible that the syllable is heard and labeled in different ways. ${ }^{3}$ It could be that when presented our visually influenced syllable, subjects hear a syllable that is unfamiliar to them but most closely approximates the familiar /va/ category. For example, it is possible that our stimulus is heard as involving a labiodental stop that, presumably, is not a familiar phonetic category for our subjects. Faced with labeling this percept, subjects would choose a syllable that is part of their native language, that is, $/ \mathrm{va} /$. Thus, the visually influenced and audiovisually compatible syllables might both induce compelling consonantal percepts (a labiodental stop and labiodental fricative, respectively). If so, then when faced with the AXB task, subjects match the audiovisual (compatible) labiodental fricative to the audio-alone labiodental fricative, regardless of the degree to which each audiovisual syllable is perceptually compelling. Future experiments will be designed to test this issue using other visually influenced stimuli.

Another interpretation of our data is that subjects did hear the visually influenced and audiovisually compatible syllables as phonetically equivalent and based their matching preferences on phonetically extraneous properties. Although Experiment 3 was designed to limit matches based on these properties by manipulating F0, it could be that an acoustic property unaffected by this manipulation (e.g., absolute onset intensity) overrode the F0 change and continued to bias matching judgments. Future experiments that manipulate other phonetically extraneous properties will help determine the validity of this interpretation.

In conclusion, the issue of the strength of the McGurk effect can now be readdressed. As mentioned in the introduction, the McGurk effect is effective in a number of highly varied identification contexts, suggesting that it is quite robust. However, the outcome of two of the discrimination experiments (Experiments 2 and 3 ) reported above suggests that the effect has its limitations. It should be noted, however, that in all three discrimination experiments, visual information did show a significant influence on matching judgments. This consistent influence suggests that the McGurk effect is robust enough to warrant further research and theorizing on audiovisual speech integration.

\section{REFERENCES}

Borden, G. J., HARRIS, K. S. (1984). Speech science primer: Physiology, acoustics, and perception of speech. Baltimore: Williams \& Wilkins.

DieHL, R. L., \& KLUENDER, K. R. (1989). On the objects of speech perception. Ecological Psychology, 1, 121-144.

Fowler, C. A., \& Rosenblum, L. D. (1991). Perception of the phonetic gesture. In I. G. Mattingly \& M. Studdert-Kennedy (Eds.), Modularity and the motor theory (pp. 33-59). Hillsdale, NJ: Erlbaum.

GreEN, K. P., \& KUHL, K. (1991). Integral processing of visual place and auditory voicing information during phonetic perception. Journal of Experimental Psychology: Human Perception \& Performance, 17, 278-288.

Green, K. P., Kuhl, P. K., Meltzoff, A. M., \& Stevens, E. B. (1991). Integrating speech information across talkers, gender, and sensory modality: Female faces and male voices in the McGurk effect Perception \& Psychophysics, 50, 524-536.

KLATt, D. H. (1980). Software for a cascade/parallel formant synthesizer. Joumal of the Acoustical Society of America, 67, 971-995. Liberman, A. M., Harris, K. S., Hoffman, H. S., \&riffith, B. C. (1957). The discrimination of the speech sound within and across phoneme boundaries. Joumal of Experimental Psychology, 54, 358-368. 
Liberman, A. M., Mattingly, I. G. (1985). The motor theory of speech perception revised. Cognition, 21, 1-36.

Massaro, D. W. (1987). Speech perception by ear and eye: A paradigm for psychological enquiry. Hillsdale, NJ: Erlbaum.

Massaro, D. W., Cohen, M. M. (1990). Perception of synthesized audible and visible speech. Psychological Science, 1, 55-63.

McGrath, M., \& Summerfield, A. Q. (1985). Intermodal timing relations and audio-visual speech recognition by normal-hearing adults. Joumal of the Acoustical Society of America, 77, 678-685.

McGurk, H., MacDonald, J. W. (1976). Hearing lips and seeing voices. Nature, 264, 746-748.

RepP, B. H., Manuel, S. Y., Liberman, A. M., \& StuddertKENNEDY, M. (1983, November). Exploring the "McGurk effect." Paper presented at the meeting of the Psychonomic Society, San Diego.

Sekiyama, K., Tohkura, Y. (1991). McGurk effect in non-English listeners: Few visual effects for Japanese subjects hearing Japanese syllables of high auditory intelligibility. Joumal of the Acoustical Society of America, 90, 1797-1805.

Silverman, K. (1987). The structure and processing of fundamental frequency contours. Unpublished doctoral dissertation, Cambridge University, Cambridge.

Summerfie LD, Q. (1987). Some preliminaries to a comprehensive account of audio-visual speech perception. In $B$. Dodd \& $R$. Campbell (Eds.), Hearing by eye: The psychology of lip reading (pp. 3-51). Hillsdale, NJ: Erlbaum.
Summerfield, Q., Macleod, P., McGrath, M.. Brooke, N. M. (1989). Lips, teeth, and the benefits of lipreading. In A. W. Young \& H. D. Ellis (Eds.), Handbook of research on face processing (pp. 223-233). Amsterdam: Elsevier.

Summerfield, Q., McGrath, M. (1984). Detection and resolution of audio-visual incompatibility in the perception of vowels. Quarterly Journal of Experimental Psychology, 36A, 51-74

\section{NOTES}

1. Acoustic dimensions that are considered extraneous to phonetic classifications in one context might be relevant in another

2. Although F0 can act as important information for phonetic characterizations (e.g., Silverman, 1987), it is assumed that changing the absolute F0 contour across tokens would have very little influence over phonetic identifications for these stimuli. This assumption is supported by the audio-alone identification results reported for Experiment 3.

3. We thank Carol Fowler for suggesting this interpretation of our results.

(Manuscript received August 22, 1991; revision accepted for publication March 10, 1992.) 